\title{
Expression of perilipin in human promyelocytic cells in response to Anaplasma phagocytophilum infection results in modified lipid metabolism
}

\author{
Correspondence \\ José de la Fuente \\ jose_delafuente@yahoo.com \\ or \\ jose.de_la_fuente@okstate.edu
}

Received 10 July 2007

Accepted 26 September 2007

\author{
Raúl Manzano-Roman, ${ }^{1}$ Consuelo Almazán, ${ }^{2}$ Victoria Naranjo, ${ }^{3}$ \\ Edmour F. Blouin, ${ }^{1}$ Katherine M. Kocan ${ }^{1}$ and José de la Fuente ${ }^{1,3}$
}

\author{
${ }^{1}$ Department of Veterinary Pathobiology, Center for Veterinary Health Sciences, Oklahoma State \\ University, Stillwater, OK 74078, USA \\ ${ }^{2}$ Facultad de Medicina Veterinaria y Zootecnia, Universidad Autónoma de Tamaulipas, Km. \\ 5 carretera Victoria-Mante, CP 87000 Cd. Victoria, Tamaulipas, Mexico \\ ${ }^{3}$ Instituto de Investigación en Recursos Cinegéticos IREC (CSIC-UCLM-JCCM), Ronda de Toledo \\ s/n, 13071 Ciudad Real, Spain
}

\begin{abstract}
The obligate intracellular pathogen Anaplasma phagocytophilum is transmitted by ticks and causes human granulocytic anaplasmosis, tick-borne fever of ruminants, and equine and canine granulocytic anaplasmosis. In a previous study, the perilipin (PLIN) gene was identified as one of the genes differentially expressed in human promyelocytic $\mathrm{HL}-60$ cells in response to infection with $A$. phagocytophilum. PLIN is a major adipocyte lipid droplet-associated phosphoprotein that plays a central role in lipolysis and cholesterol synthesis. Host cholesterol and other lipids are required by $A$. phagocytophilum for infection and multiplication in human cells. In this study, it was hypothesized that PLIN may be involved in infection of human HL-60 cells by $A$. phagocytophilum. To test this hypothesis, a combination of real-time RT-PCR, immunofluorescence and RNA interference was used to study the expression of PLIN. The results of these studies demonstrated that $A$. phagocytophilum modulates lipid metabolism by increasing PLIN mRNA levels and facilitates infection of HL-60 cells. The results of these studies expand our knowledge of the role of lipid metabolism in A. phagocytophilum infection and multiplication in HL-60 cells and suggest a mechanism by which $A$. phagocytophilum modulates lipid metabolism.
\end{abstract}

\section{INTRODUCTION}

Anaplasma phagocytophilum (Rickettsiales: Anaplasmataceae) is an obligate intracellular tick-borne pathogen that causes human granulocytic anaplasmosis (HGA), tickborne fever of ruminants, and equine and canine granulocytic anaplasmosis (Dumler et al., 2001). HGA, first described in 1994 in the United States, has become a predominant form of anaplasmosis and is among the most common tick-borne diseases in the United States and Europe (Dumler et al., 2005).

A. phagocytophilum initiates infection by adhering to polymorphonuclear leukocytes, a process which involves adhesins such as the human P-selectin glycoprotein ligand1 (PSGL-1) that bind cooperatively to neutrophil ligand molecules (Dumler et al., 2005). Host cholesterol is incorporated into the A. phagocytophilum membrane and is required for pathogen infection and multiplication, involving cholesterol-rich lipid drafts or caveolae and

Abbreviations: p.i., post-infection; PLIN, perilipin; PSGL-1, P-selectin glycoprotein ligand-1; RNAi, RNA interference. glycosylphosphatidylinositol-anchored proteins (Lin \& Rikihisa, 2003a, b). After infection, A. phagocytophilum undergoes a developmental cycle within a parasitophorous vacuole in which reticulated forms divide by binary fission and then transform into the infective dense forms. This infection cycle modulates host cell growth and differentiation (Carlyon \& Fikrig, 2003).

While the main vectors for $A$. phagocytophilum are tick species belonging to the Ixodes ricinus complex, the pathogen multiplies in a broad range of terrestrial vertebrates (Dumler et al., 2005; de la Fuente et al., 2005a). In the laboratory, A. phagocytophilum can be propagated in the undifferentiated human promyelocytic cell line HL-60. Infection of HL-60 cells with $A$. phagocytophilum results in modulation of host cell gene expression (see, for example, de la Fuente et al., 2005b; Pedra et al., 2005; Sukumaran et al., 2005).

In a previous study, we identified the perilipin (PLIN) gene as one of the genes differentially expressed in human HL60 cells in response to infection with A. phagocytophilum (de la Fuente et al., 2005b). PLIN is a major adipocyte lipid 
droplet-associated phosphoprotein that plays a central role during lipolysis and cholesterol synthesis (Yeaman, 2004; Moore et al., 2005; Miyoshi et al., 2006; Granneman et al., 2007). Because of the key role of cholesterol and other lipids in A. phagocytophilum infection and multiplication of human cells, we hypothesized that PLIN may be involved in the infection of human cells with A. phagocytophilum. We tested this hypothesis in the HL-60 human cell line infected with $A$. phagocytophilum using a combination of real-time RT-PCR, immunofluorescence and RNA interference (RNAi).

\section{METHODS}

Determination of PLIN mRNA levels in uninfected and infected HL-60 cells. The human HL-60 cell line was cultured and infected with A. phagocytophilum as previously described (de la Fuente et al., 2005b). Three independent uninfected and infected cultures were sampled at $0,12,24,48,72$ and 96 h post-infection (p.i.) and human PLIN and A. phagocytophilum major surface protein 4 (msp4) mRNA levels were determined by real-time RT-PCR using human PLIN (GenBank accession no. NM_002666) and msp4 (de la Fuente et al., 2005a) sequence-specific primers (PLIN, PLINRT5:5'-CTCTCGATACACCGTGCAGA and PLINRT3: $5^{\prime}$-TGGTCCTCATGATCCTCCTC; msp4, APMSP4RT5: $5^{\prime}$-TGACAGGGGAGGATCTTACG and APMSP4RT3:5'-TCTAGCTCCGCCAATAGCAT) and the QuantiTec SYBR Green RT-PCR kit (Qiagen) in a Bio-Rad iQ5 thermal cycler following the manufacturer's recommendations. mRNA levels were normalized against human $\beta$-actin (forward: $5^{\prime}$ TGATATCGCCGCGCTCGTCGTC; reverse: 5'-GCCGATCCACACGGAGTACT; de la Fuente et al., 2005b) and displayed in mRNA arbitrary units. PLIN mRNA levels were compared in infected and uninfected cells using the ANOVA test $(P=0.05)$.

PLIN immunofluorescent labelling in uninfected and infected HL-60 cells. Smears of uninfected and A. phagocytophilum-infected HL-60 cells collected at $124 \mathrm{~h}$ p.i. were prepared using a cytocentrifuge. The cells were dried and fixed immediately with $4 \%$ paraformaldehyde in PBS for $20 \mathrm{~min}$ at room temperature (RT). The cells were permeabilized with $0.2 \%$ Triton X-100 in PBS for 20 min and blocked with $2.5 \%$ BSA in PBS for 1 h. The cells were then incubated with rabbit polyclonal anti-perilipin antibodies $(1: 10)$ (Novus Biologicals), rabbit polyclonal antibodies to A. phagocytophilum $(1: 200)$ (de la Fuente et al., 2006) or rabbit preimmune control serum $(1: 200)$ for $1 \mathrm{~h}$ at RT. The cells were then incubated with Cy3 or Cy5 goat anti-rabbit IgG $(1: 100)$ secondary antibodies (Jackson Immuno Research) for $1 \mathrm{~h}$ in the dark at RT. Between each step, the slides were rinsed three times with PBS for 3 min each and kept in PBS before observing them on a confocal microscope (Leica). During all incubations, the cells were rocked on a LabQuake shaker (Barnstead/Thermolyne).

Determination of total cholesterol content in uninfected and infected HL-60 cells. Uninfected and A. phagocytophilum-infected HL-60 cells were collected at 72, 120, 144 and $160 \mathrm{~h}$ p.i. and the total cholesterol levels were determined using the Amplex Red cholesterol assay kit according to the manufacturer's instructions (Molecular Probes). Total cholesterol content was determined with a Polarstar Optima spectrophotometer (BMG Lab Technologies) and normalized against the total protein concentration as determined by the DC Protein Assay (Bio-Rad). Three replicates were conducted for each time point and the cholesterol content was compared in infected and uninfected cells using the Student's $t$-test $(P=0.05)$.

RNA interference in HL-60 cells. Gene expression was silenced in HL-60 cells by RNAi using a combination of two different predesigned siRNAs to PLIN (NM_002666; siRNA IDs 120985 and 120986) and to actin-related protein 3 (ARP3) (NM_020445; siRNA IDs 127242 and 127243) and PSGL-1 (NM_003006; siRNAs IDs 12441 and 142575) to serve as negative and positive controls, respectively (Ambion). In a 96-well plate, $4 \times 10^{5}$ cells per well were nucleofected with $1 \mu \mathrm{g}$ siRNA using the Nucleofector 96-well shuttle system (Amaxa Biosystems) with kit SF and program 96-EN-138 following the manufacturer's instructions (efficiency of transfection $71 \pm 19 \%$ after $24 \mathrm{~h}$ ). After nucleofection, cells were divided into two 96-well plates. Twenty-four hours after nucleofection, cells were collected from one plate for assessment of viability and morphology in Giemsa-stained cytospin smears, RNA extraction (RNeasy 96 kit; Qiagen) and analysis of gene expression by real-time RT-PCR as described above. The second plate was incubated for $24 \mathrm{~h}$ with cellfree A. phagocytophilum (m.o.i. $=10)$ prepared as described by Thomas \& Fikrig (2007), and the level of infection corresponded to approximately $72 \mathrm{~h}$ p.i. as depicted in Fig. 1. The cells were then washed three times with PBS and total DNA was extracted (Wizard

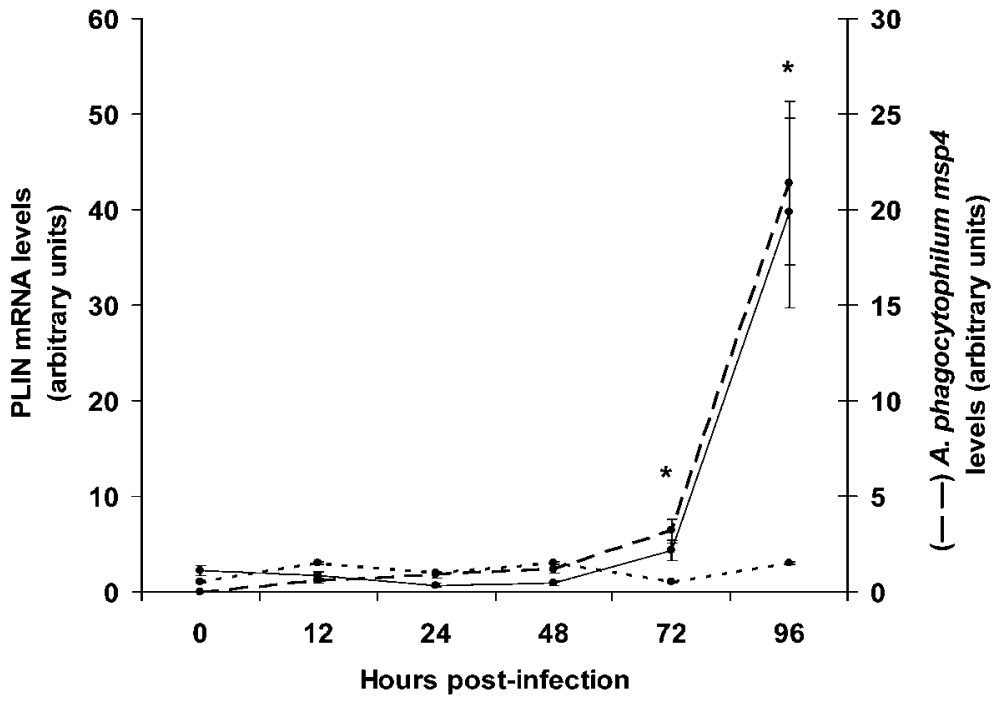

Fig. 1. Expression kinetics of PLIN in human HL-60 cells infected with $A$. phagocytophilum. Human PLIN (solid and dotted lines) and $A$. phagocytophilum msp4 (dashed line) mRNA levels were determined by real-time RT-PCR in uninfected and infected HL-60 cells. Amplification efficiencies were normalized against human $\beta$-actin and displayed in mRNA arbitrary units. PLIN mRNA levels were compared in infected (solid line) and uninfected (dotted line) cells using an ANOVA test ( ${ }^{\star} P$ $<0.05 ; n=3)$. 
SV 96 genomic DNA purification system; Promega). The $A$. phagocytophilum infection levels in HL-60 cells were evaluated after RNAi by real-time PCR of $m s p 4$ and normalizing against human Alu sequences (Nicklas \& Buel, 2003) using the QuantiTec SYBR Green PCR kit (Qiagen) in an iQ5 thermal cycler (Bio-Rad) as described previously. Known amounts of the full-length A. phagocytophilum msp4 PCR were used to construct a standard curve for the real-time PCR. PLIN and PSGL-1 mRNA levels were determined after RNAi by real-time RT-PCR, normalized against human $\beta$-actin using the comparative Ct (delta delta $\mathrm{Ct}$ ) method and compared in PLIN or PSGL-1 siRNA- and ARP3 siRNA-treated control cells using the Student's $t$-test $(P=0.05 ; n=4-8) . A$. phagocytophilum msp4 DNA levels were compared in cells nucleofected with PLIN or PSGL-1 siRNAs and control cells treated with ARP3 siRNA using the Student's $t$-test $(P=0.05 ; n=4-8)$.

\section{RESULTS AND DISCUSSION}

In a previous study in which differential gene expression of HL-60 cells in response to A. phagocytophilum infection was characterized by microarray analysis, we demonstrated that expression of PLIN was induced at $72 \mathrm{~h}$ p.i. (de la Fuente et al., 2005b). Because of the important role of PLIN during lipolysis and cholesterol synthesis (Yeaman, 2004; Moore et al., 2005; Miyoshi et al., 2006; Granneman et al., 2007), which are essential for A. phagocytophilum infection and multiplication (Lin \& Rikihisa, 2003a, b; Xiong et al., 2007), we analysed the expression of PLIN at the mRNA and protein levels in uninfected and $A$. phagocytophilum-infected HL-60 cells.

The PLIN mRNA levels increased in HL-60 cells after $48 \mathrm{~h}$ p.i. with A. phagocytophilum and reached 37 -fold induction at $96 \mathrm{~h}$ p.i. (Fig. 1). The increase in PLIN mRNA levels coincided with pathogen multiplication and increasing infection levels (Fig. 1). PLIN protein levels were higher in A. phagocytophilum-infected HL-60 cells than in uninfected control cells (Fig. 2a-h). Similar to results in adipocytes (Moore et al., 2005; Miyoshi et al., 2006), PLIN was localized in the cytoplasm and in the periphery of infected HL-60 cells (Fig. 2h), where A. phagocytophilum labelling was also found in some cells (see insert on Fig. $2 \mathrm{~g}$ ). In the adipocytes, PLIN preferentially targets a special class of peripheral lipid storage droplets, but not the major or central lipid storage droplets (Moore et al., 2005).

The increase of PLIN mRNA and protein levels in $A$. phagocytophilum-infected HL-60 cells may reflect a protective cellular response to limit rickettsial infection or may be the result of the manipulation by A. phagocytophilum of host gene expression to promote pathogen multiplication. To test these hypotheses, the effect of PLIN knockdown by RNAi was evaluated in HL-60 cells infected with $A$. phagocytophilum. After RNAi, expression of PLIN and PSGL-1 was shown to be reduced by $51 \pm 14 \%(n=4)$ and

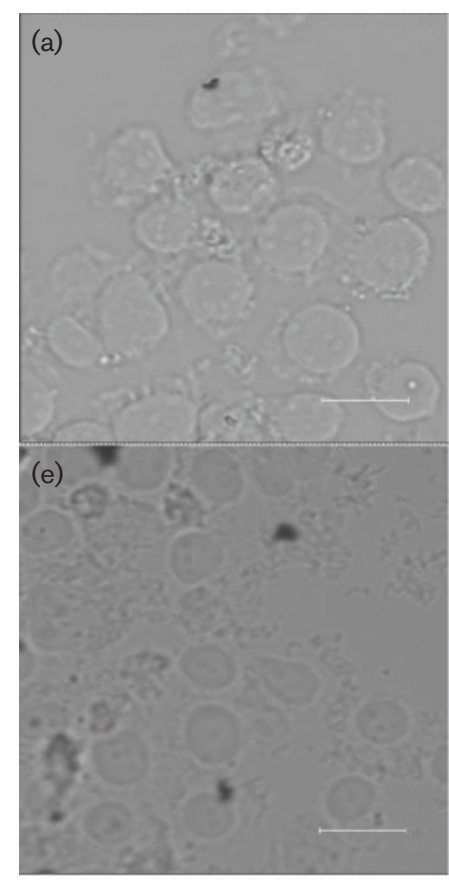

White field
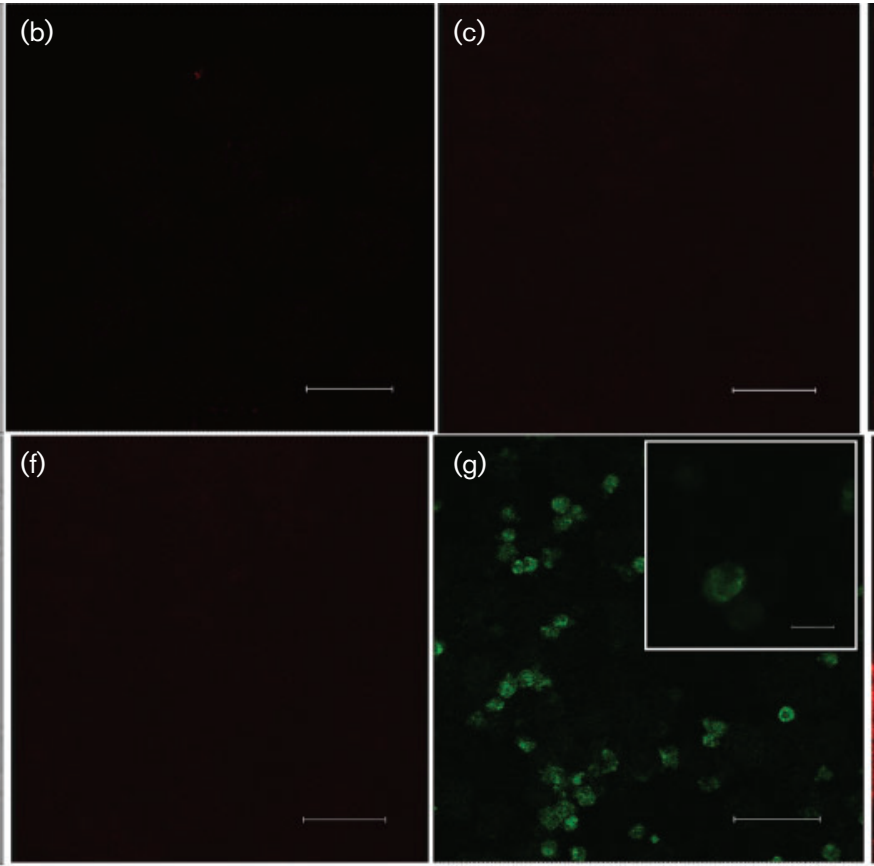

Control serum
Anti-A. phagocytophilum
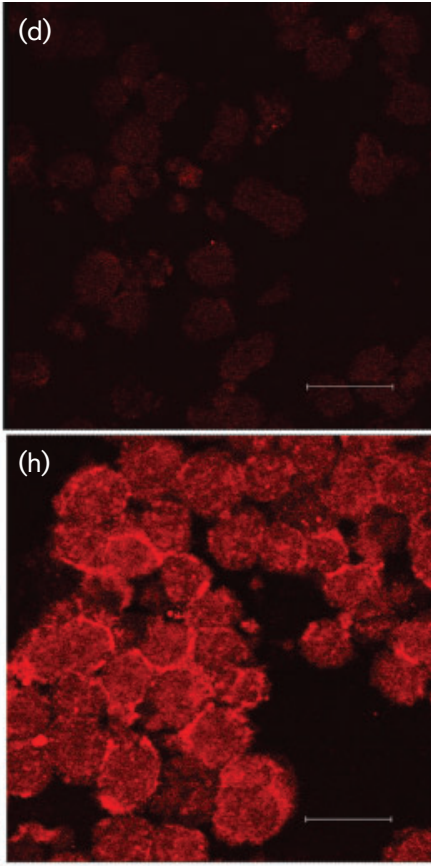

Anti-PLIN

Fig. 2. Immunofluorescent labelling for PLIN and $A$. phagocytophilum in uninfected and infected human HL-60 cells. Uninfected $(a-d)$ and infected $(e-h) H L-60$ cells were incubated with rabbit preimmune control serum (b, $f)$ or anti- $A$. phagocytophilum (c, g) or anti-PLIN (d, h) antibodies. Scale bars, $29.88 \mu \mathrm{m}(\mathrm{a}, \mathrm{b}), 50 \mu \mathrm{m}(\mathrm{c}, \mathrm{e}, \mathrm{f}), 61.89 \mu \mathrm{m}(\mathrm{d}), 88.92 \mu \mathrm{m}(\mathrm{g}$; insert, $21.61 \mu \mathrm{m})$ and $40.94 \mu \mathrm{m}(\mathrm{h})$. 
$61 \pm 18 \%(n=8)$, respectively, as compared with the ARP3 siRNA-nucleofected controls $(P<0.05)$. RNAi also resulted in a reduction in $A$. phagocytophilum DNA in cells nucleofected with PLIN siRNA, similar to results obtained in positive control cells transfected with PSGL-1 siRNA (Fig. 3). If PLIN has a role in the control of $A$. phagocytophilum infection in humans, we would have expected higher infection levels in HL-60 cells with knockdown PLIN. However, the lower infection levels that resulted after RNAi suggested that PLIN was required for A. phagocytophilum infection and/or multiplication in HL60 cells.

A. phagocytophilum infection and multiplication involve cholesterol-rich lipid drafts or caveolae and glycosylphosphatidylinositol-anchored proteins (Lin \& Rikihisa, 2003a). Because A. phagocytophilum lacks genes for lipid A and peptidoglycan biosynthesis, the pathogen requires the incorporation of host cholesterol into the membrane for survival (Lin \& Rikihisa, 2003b). Furthermore, a highcholesterol diet facilitates $A$. phagocytophilum infection of apolipoprotein E-deficient mice (Xiong et al., 2007). These results are in agreement with the findings reported herein and support a role for PLIN during infection and multiplication of $A$. phagocytophilum in human host cells.

The overexpression of host PLIN in A. phagocytophiluminfected HL-60 cells suggested a mechanism by which lipolysis and cholesterol synthesis are affected during pathogen infection and multiplication in human cells. The hormone-sensitive lipase (HSL) catalyses the forma-

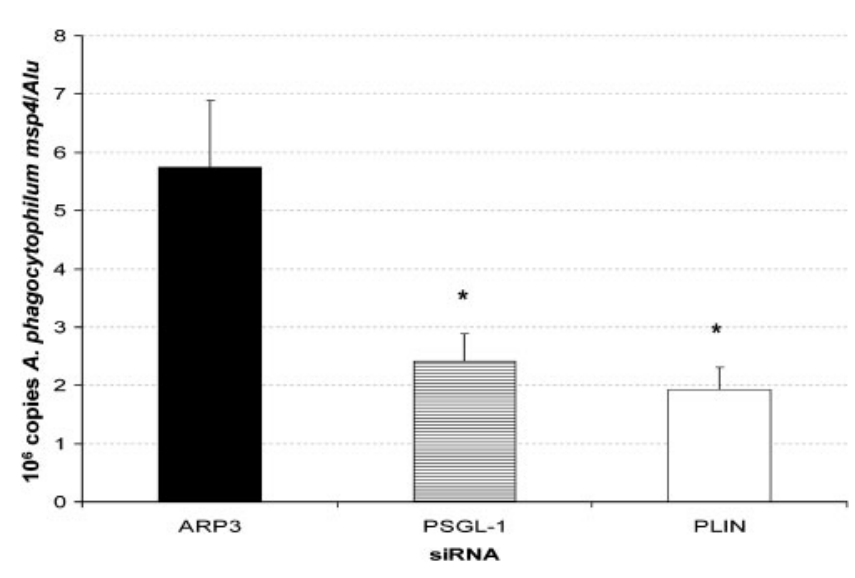

Fig. 3. Effect of PLIN on A. phagocytophilum infection of human $\mathrm{HL}-60$ cells. $A$. phagocytophilum msp4 DNA levels were determined by real-time PCR in infected HL-60 cells with knockdown PLIN, PSGL-1 (positive control) and ARP3 (negative control). A. phagocytophilum msp4 DNA levels were normalized against human $A / u$ sequences and infection levels were compared in cells nucleofected with PLIN or PSGL-1 siRNAs and control cells treated with ARP3 siRNA by Student's $t$-test $\left({ }^{\star} P<0.05\right.$; $n=4-8)$. tion of cholesterol in macrophages and other cells (Yeaman, 2004). Although contradictory results have been reported on the role of PLIN in promoting or preventing lipolysis (Yeaman, 2004), PLIN was recently shown to promote HSL-mediated adipocyte lipolysis (Moore et al., 2005; Miyoshi et al., 2006; Granneman et al., 2007). Congruent with these findings, the total cholesterol content was higher in A. phagocytophilum-infected cells collected at 72 and $120 \mathrm{~h}$ p.i. than in uninfected HL-60 cells (Fig. 4). At 144 and 160 h p.i., cholesterol levels were similar in infected and uninfected cells, presumably due to incorporation into the bacterial cells (Lin \& Rikihisa, 2003b).

\section{Conclusions}

In summary, we have shown that A. phagocytophilum infection of human promyelocytic HL-60 cells results in increased PLIN mRNA and protein levels. These results have expanded our knowledge of the important role that lipid metabolism plays in A. phagocytophilum infection and multiplication and suggest a new mechanism by which $A$. phagocytophilum modulates lipid metabolism by promoting HSL-mediated lipolysis in infected cells. Furthermore, silencing of PLIN expression reduced pathogen infection/ multiplication, thus suggesting that PLIN is not induced in host cells as a defence mechanism to limit pathogen multiplication but rather A. phagocytophilum modulates the expression of PLIN to facilitate host cell infection. Further studies are needed to define the mechanism by which A. phagocytophilum modulates the expression of PLIN in human HL-60 cells. Nonetheless, the results of these studies suggest that therapeutics and other interventions that target host lipid metabolism will likely aid in the control of A. phagocytophilum infection in humans and animals (Xiong et al., 2007).

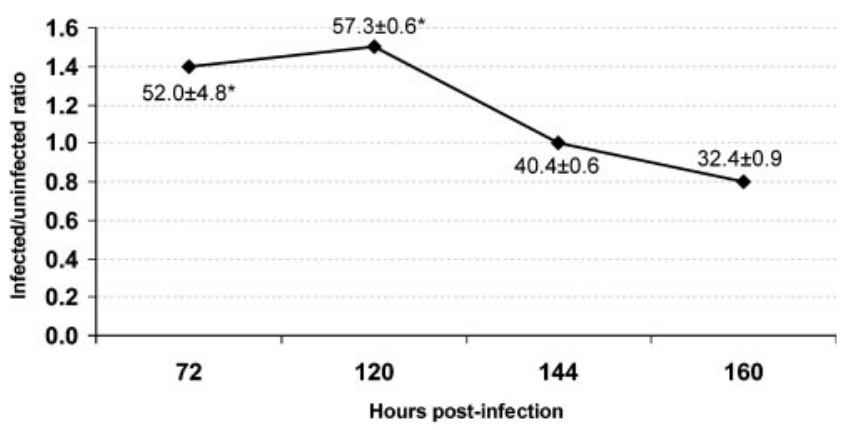

Fig. 4. Total cholesterol content $\left[\mu \mathrm{g}(\mathrm{mg} \text { protein })^{-1}\right]$ in $A$. phagocytophilum-infected and uninfected HL-60 cells. Total cholesterol content was determined in uninfected [39.4 $\pm 4.5 \mu \mathrm{g}$ (mg protein $)^{-1}$ ] and infected cells at $72,120,144$ and $160 \mathrm{~h} \mathrm{p.i.}$ (shown in the graph), normalized against total protein concentration and compared by Student's $t$-test $\left({ }^{\star} P \leqslant 0.01 ; n=3\right.$ for each time point). 


\section{ACKNOWLEDGEMENTS}

This research was supported by the Oklahoma Agricultural Experiment Station (Project 1669), the Walter R. Sitlington Endowed Chair for Food Animal Research (K.M.K.) and the Ministry of Science and Education (MEC), Spain, (project AGL2005-07401). R.M.-R. was funded by the Ministerio de Educación y Ciencia, Spain. V.N. was funded by the Consejería de Educación, JCCM, Spain.

\section{REFERENCES}

Carlyon, J. A. \& Fikrig, E. (2003). Invasion and survival strategies of Anaplasma phagocytophilum. Cell Microbiol 5, 743-754.

de la Fuente, J., Massung, R. F., Wong, S. J., Chu, F. K., Lutz, H., Meli, M., von Loewenich, F. D., Grzeszczuk, A., Torina, A. \& other authors (2005a). Sequence analysis of the msp4 gene of Anaplasma phagocytophilum strains. J Clin Microbiol 43, 1309-1317.

de la Fuente, J., Ayoubi, P., Blouin, E. F., Almazán, C., Naranjo, V. \& Kocan, K. M. (2005b). Gene expression profiling of human promyelocytic cells in response to infection with Anaplasma phagocytophilum. Cell Microbiol 7, 549-559.

de la Fuente, J., Almazán, C., Blouin, E. F., Naranjo, V. \& Kocan, K. M. (2006). Reduction of tick infections with Anaplasma marginale and $A$. phagocytophilum by targeting the tick protective antigen subolesin. Parasitol Res 100, 85-91.

Dumler, J. S., Barbet, A. C., Bekker, C. P. J., Dasch, G. A., Palmer, G. H., Ray, S. C., Rikihisa, Y. \& Rurangirwa, F. R. (2001). Reorganization of the genera in the families Rickettsiaceae and Anaplasmataceae in the order Rickettsiales: unification of some species of Ehrlichia with Anaplasma, Cowdria with Ehrlichia and Ehrlichia with Neorickettsia, descriptions of six new species combinations and designation of Ehrlichia equi and 'HGE agent' as subjective synonyms of Ehrlichia phagocytophila. Int J Syst Evol Microbiol 51, 2145-2165.

Dumler, J. S., Choi, K. S., Garcia-Garcia, J. C., Barat, N. S., Scorpio, D. G., Garyu, J. W., Grab, D. J. \& Bakken, J. S. (2005). Human granulocytic anaplasmosis and Anaplasma phagocytophilum. Emerg Infect Dis 11, 1828-1834.
Granneman, J. G., Moore, H. P., Granneman, R. L., Greenberg, A. S., Obin, M. S. \& Zhu, Z. (2007). Analysis of lipolytic protein trafficking and interactions in adipocytes. J Biol Chem 282, 5726-5735.

Lin, M. \& Rikihisa, Y. (2003a). Obligatory intracellular parasitism by Ehrlichia chaffeensis and Anaplasma phagocytophilum involves caveolae and glycosylphosphatidylinositol-anchored proteins. Cell Microbiol 5, 809-820.

Lin, M. \& Rikihisa, Y. (2003b). Ehrlichia chaffeensis and Anaplasma phagocytophilum lack genes for lipid A biosynthesis and incorporate cholesterol for their survival. Infect Immun 71, 5324-5331.

Miyoshi, H., Souza, S. C., Zhang, H. H., Strissel, K. J., Christoffolete, M. A., Kovsan, J., Rudich, A., Kraemer, F. B., Bianco, A. C. \& other authors (2006). Perilipin promotes hormone-sensitive lipasemediated adipocyte lipolysis via phosphorylation-dependent and independent mechanisms. J Biol Chem 281, 15837-15844.

Moore, H. P., Silver, R. B., Mottillo, E. P., Bernlohr, D. A. \& Granneman, J. G. (2005). Perilipin targets a novel pool of lipid droplets for lipolytic attack by hormone-sensitive lipase. J Biol Chem 280, 43109-43120.

Nicklas, J. A. \& Buel, E. (2003). Development of an Alu-based, realtime PCR method for quantitation of human DNA in forensic samples. J Forensic Sci 48, 936-944.

Pedra, J. H., Sukumaran, B., Carlyon, J. A., Berliner, N. \& Fikrig, E. (2005). Modulation of NB4 promyelocytic leukemic cell machinery by Anaplasma phagocytophilum. Genomics 86, 365-377.

Sukumaran, B., Carlyon, J. A., Cai, J. L., Berliner, N. \& Fikrig, E. (2005). Early transcriptional response of human neutrophils to Anaplasma phagocytophilum infection. Infect Immun 73, 8089-8099.

Thomas, V. \& Fikrig, E. (2007). Anaplasma phagocytophilum specifically induces tyrosine phosphorylation of ROCK1 during infection. Cell Microbiol 9, 1730-1737.

Xiong, Q., Wang, X. \& Rikihisa, Y. (2007). High-cholesterol diet facilitates Anaplasma phagocytophilum infection and up-regulates macrophage inflammatory protein-2 and CXCR2 expression in apolipoprotein E-deficient mice. J Infect Dis 195, 1497-1503.

Yeaman, S. J. (2004). Hormone-sensitive lipase - new roles for an old enzyme. Biochem J 379, 11-22. 